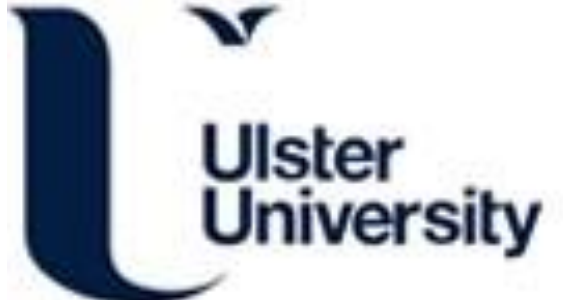

\section{A Multivariate Empirical Mode Decomposition based Filtering for Subject Independent $\mathrm{BCl}$}

Gaur, P., Pachori, RB., Wang, H., \& Prasad, G. (2016). A Multivariate Empirical Mode Decomposition based Filtering for Subject Independent BCI. In Unknown Host Publication IEEE Xplore. http://uir.ulster.ac.uk/37251/2/FW\%20ISSC\%202016\%20review\%20decision.txt

Link to publication record in Ulster University Research Portal

\section{Published in:}

Unknown Host Publication

Publication Status:

Published online: 04/08/2016

\section{Document Version \\ Author Accepted version}

\section{General rights}

Copyright for the publications made accessible via Ulster University's Research Portal is retained by the author(s) and / or other copyright owners and it is a condition of accessing these publications that users recognise and abide by the legal requirements associated with these rights.

\section{Take down policy}

The Research Portal is Ulster University's institutional repository that provides access to Ulster's research outputs. Every effort has been made to ensure that content in the Research Portal does not infringe any person's rights, or applicable UK laws. If you discover content in the Research Portal that you believe breaches copyright or violates any law, please contact pure-support@ulster.ac.uk. 


\title{
A Multivariate Empirical Mode Decomposition based Filtering for Subject Independent BCI
}

\author{
Pramod Gaur*, Ram Bilas Pachori ${ }^{\dagger}$, Hui Wang ${ }^{\ddagger}$ and Girijesh Prasad* \\ *Intelligent Systems Research Centre, Ulster University, L'Derry, U.K. e-mail: gaur-p@email.ulster.ac.uk; g.prasad@ulster.ac.uk \\ ${ }^{\dagger}$ Discipline of Electrical Engineering, Indian Institute of Technology Indore, Indore, India. e-mail: pachori@iiti.ac.in \\ ${ }_{\ddagger}^{\ddagger}$ School of Computing and Mathematics, Ulster University, Jordanstown, U.K. e-mail:h.wang@ulster.ac.uk
}

\begin{abstract}
Goal: A brain-computer interface (BCI) provides a way to translate the motion intentions of human using brain signals such as electroencephalogram (EEG) into control commands. EEG signals are highly subject specific and non-stationary. One of the most challenging tasks is to classify motion intentions since the recorded EEG signals have inherent non-stationarities which are due to changes in the signal properties over time within as well as across sessions. Thus it becomes difficult to achieve a stable operation of BCI. Method: We present a novel filtering method based on the multivariate empirical mode decomposition (MEMD) using subject independent BCI (MEMD-SI-BCI) for classification of motor imagery (MI) based EEG signals to achieve enhanced BCI. A subject independent BCI can be used immediately by the new user without using the user's training data. The MEMD method helps to utilize the cross channel information and enhance localization properties. It decomposes multichannel EEG signals into a set of multivariate intrinsic mode functions (MIMFs). These MIMFs can be considered narrowband, amplitude and frequency modulated (AM-FM) signals. The statistical property, namely, mean frequency measure of these MIMFs has been used to combine these MIMFs to compute the enhanced EEG signals which have major contributions due to $\mu$ and $\beta$ rhythms over the motor cortex region. The objective of the proposed method is to filter EEG signals before feature extraction and classification to enhance the features separability and ultimately the BCI task classification performance. The common spatial pattern (CSP) feature has been computed from the enhanced EEG signals and has been used as a feature set for classification of left hand and right hand MIs using a linear discriminant analysis (LDA) based classification method. Results: We have achieved an improvement of $>11 \%$ in the evaluation stage using the MEMD-SI-BCI method when compared with SI-BCI. Significance: This study helps to develop BCI systems with intuitive motor imaginations, thus facilitates broad use of noninvasive BCIs. We have evaluated our method on publicly available BCI competition IV dataset $2 \mathrm{~A}$ and have obtained improved performance.
\end{abstract}

Index Terms-Brain-computer interface (BCI); empirical mode decomposition (EMD); common spatial pattern (CSP); linear discriminant analysis (LDA) classifier.

\section{INTRODUCTION}

A brain-computer interface (BCI) provides an alternate means of communication for people suffering from neurological impairments [1]. It enables them to interact with the external environment by sending commands to computer using their brain activity measured by e.g. electroencephalogram (EEG) signals. It has worked as promising tool for healthy people and disabled people such as P300 spellers and video games [2]. The BCI technology has many applications in the biomedical engineering and neuroprosthetics [3], [4]. In neurophysiology, motor intention shows enhancement (ERS: Event-Related Synchronization) or attenuation (ERD: EventRelated Desynchronization) of rhythmic activity in the specific frequency bands of $\mu$ rhythm $(8-13 \mathrm{~Hz})$ and $\beta$ rhythm $(14-30$ $\mathrm{Hz}$ ) over the sensorimotor cortex [1], [5]. This phenomenon is known as motor imagery (MI) response in frequency bands. It can be explained as the mental rehearsal of a motor act without actual execution of movement [1]. In BCI research community, EEG based BCI has received attention due to high temporal resolution, the ease of use and low cost when compared to other non-invasive techniques available for measuring brain activity, such as Magnetoencephalography (MEG), Positron emission tomography (PET) scans. But, it suffers from serious challenges such as non-stationarity, low signal to noise ratio (SNR), highly subject specific data [6], [7] and artifacts such as electrooculography (EOG) and electromyography (EMG) and power line. The inherent non-stationarity present in the recorded EEG signals makes the classification of motion intensions one of the demanding tasks. Another major issue of MI based BCI is that they are subject specific. The process involves recording of EEG signals and training for each of the new subject, which is very time consuming process.

Recently, empirical mode decomposition (EMD) based filtering has been proposed [8] using the mean frequency measure of intrinsic mode functions (IMFs) in order to obtain the enhanced EEG signals for BCI. Multivarite version of EMD (MEMD) has been studied [9] to account for inherent non-stationarity and utilize correlation information present in multichannel EEG signals. The technique exploring the selforganizing fuzzy neural network has been studied in order to achieve the higher classification accuracy in MI tasks in motor imagery based brain computer interface (MI-BCI) [10]. This paper extends our previously proposed single channel EMD based filtering and presents the design of a novel multi channel MEMD filtering based subject independent (SI)-BCI by training the system on EEG data from multiple subjects. The trained model has been used to solve the two-class classification problem, namely, left and right hand MIs. It should be noted that the MEMD method is adaptive data driven method by nature and highly suitable for analysis of nonlinear and non-stationary signals like EEG. It provides a set 
of IMFs which can be considered as narrow-band amplitude and frequency modulated (AM-FM) signals. The process is two-fold, first we combine all of the data from several users to create training set and then apply MEMD based filtering to utilize the cross channel information present in the channels. There are several variant of common spatial pattern (CSP) which have been explored by different research groups [11], [12]. The mean frequency of these IMFs is used to obtain enhanced EEG signals corresponding to $\mu$ and $\beta$ rhythms. The CSP features have been computed from the enhanced EEG signals. Further, a linear discriminant analysis (LDA) classifier has been used to classify the feature set into left and right hand MIs. A block diagram depicts the proposed method in Fig.1. The remainder of the paper is organized as follows: Section II describes the details about the BCI competition IV dataset 2A. Section III presents the brief introduction of MEMD. Section IV discusses about the CSP feature. Section V provides details of the LDA classifier and section VI discusses about the results obtained using the proposed methodology and section VII concludes this paper.

\section{BCI COMPETITION IV DATASET 2A DESCRIPTION}

The proposed method has been investigated on the BCI competition IV dataset 2A [13]. This dataset consists of EEG signals performing four different motor imagery tasks: movements of the left hand, right hand, feet, and tongue from nine healthy subjects. The dataset contains two sessions, one for training and one for evaluation. The sessions were recorded on different days for each of the subjects. Each session was recorded with 22 EEG channels and 3 monopolar EOG channels (with left mastoid serving as reference) and includes 288 trials of data $(72$ for each of the four motor imagery tasks). The EEG signals were bandpass filtered between 0.5 $\mathrm{Hz}$ and $100 \mathrm{~Hz}$ and sampled at the sampling rate of $250 \mathrm{~Hz}$. An additional $50 \mathrm{~Hz}$ notch filter has been applied to suppress line noise. The time interval selection for the motor imagery classification is a key factor that helps us to reduce the error rates. In this paper, we have extracted CSP feature from the enhanced EEG signals from fifteen channels as shown Fig. 2 between $0.5 \mathrm{~s}$ and $3 \mathrm{~s}$ after onset of the visual cue in the training step, where as competition winner [11] extracted from $0.5 \mathrm{~s}$ and $2.5 \mathrm{~s}$ after onset of the visual cue in the training step. Refer to Tangermann et al. [13] for further details on the BCI competition IV dataset $2 \mathrm{~A}$.

\section{Multivariate Empirical Mode Decomposition (MEMD): A REVIEW}

EEG signals tend to have low SNR and may suffer from interference from EMG, EOG, or electrosurgical units (ESUs) [1]. The signals of interest corresponding to $\mu$ and $\beta$ rhythms may have contaminating noise in the EEG signal, may cause erroneous results. Hence, a method is required that can filter out noise and does not undermine the original signal. Huang et al. proposed EMD [14], that can decompose the original signal into multiple IMFs, expressed as follows:

$$
X(t)=\sum_{j=1}^{m} C_{j}(t)+R S_{m}(t)
$$

where $X(t)$ is the original signal in time domain, $C_{j}(t)$ is $j^{t h}$ IMF, and $R S_{m}(t)$ is the the residue. Hence, we can select the IMFs combination to re-construct the signal of our interest and then, discard the remaining IMFs which contribute to noise and other artifacts. However, single channel EMD suffers from the mode mixing problem. An ensemble empirical mode decomposition (EEMD) technique has been proposed in [15] to overcome this problem. Unfortunately, EEMD is a time-consuming method and may add noise into the original signal. Further, Rehman and Mandic [9] have proposed an multivariate version of improved EMD method utilizing the cross channel information called MEMD. Later, in 2013 they proposed a noise-assisted MEMD (N-A MEMD) method [16] , which is not only suitable for dealing with multichannel signals, but also solves the problem of mode mixing using white Gaussian noise added to different channels. Hence, the N-A MEMD method has been used in this paper. In computation of N-A MEMD, the mean $M(t)$ is calculated by means of the multivariate envelope curves, expressed as follows [16]:

$$
M(t)=\frac{1}{P} \sum_{P=1}^{K} e^{\theta_{P}}(t)
$$

where $e^{\theta_{P}}(t)$ are the multivariate envelope curves for whole set of direction vectors and $P$ is length of the vectors. Then, we compute the candidate IMF $R(t)$ by $R(t)=X(t)-M(t)$. If the candidate IMF satisfies the stoppage criterion, the candidate IMF becomes the multivariate IMF. If not, the input $X(t)$ will equal the remainder $R(t)$ and compute the remainder again. The whole process is repeated until all of the multivariate IMFs are extracted. Regarding the stoppage criterion, it is similar as in the original EMD proposed by Huang et al. [14] using decomposing signal until the signal becomes monotonic.

\section{COMmon Spatial Pattern (CSP)}

In this stage, the most widely used feature corresponding to motor imagery (MI) based BCI has been extracted using CSP algorithm from fifteen channels as shown in Fig. 2. It aims at learning spatial filters which maximizes the variance of spatially filtered signals in one mental imagery task and simultaneously, minimizes the variance for other mental imagery task. The recorded EEG scalp potentials tend to have very poor spatial resolution because of volume conduction. The classification of EEG signals becomes difficult, if other sources produce strong signals and the signal of interest is weak in the specific frequency range [17].

As discussed in introduction, the CSP algorithm has shown promising results in calculating spatial filters for detecting (ERD/ERS) [17],[11]. It is a data-driven supervised decomposition of signals parameterized by a projection matrix 


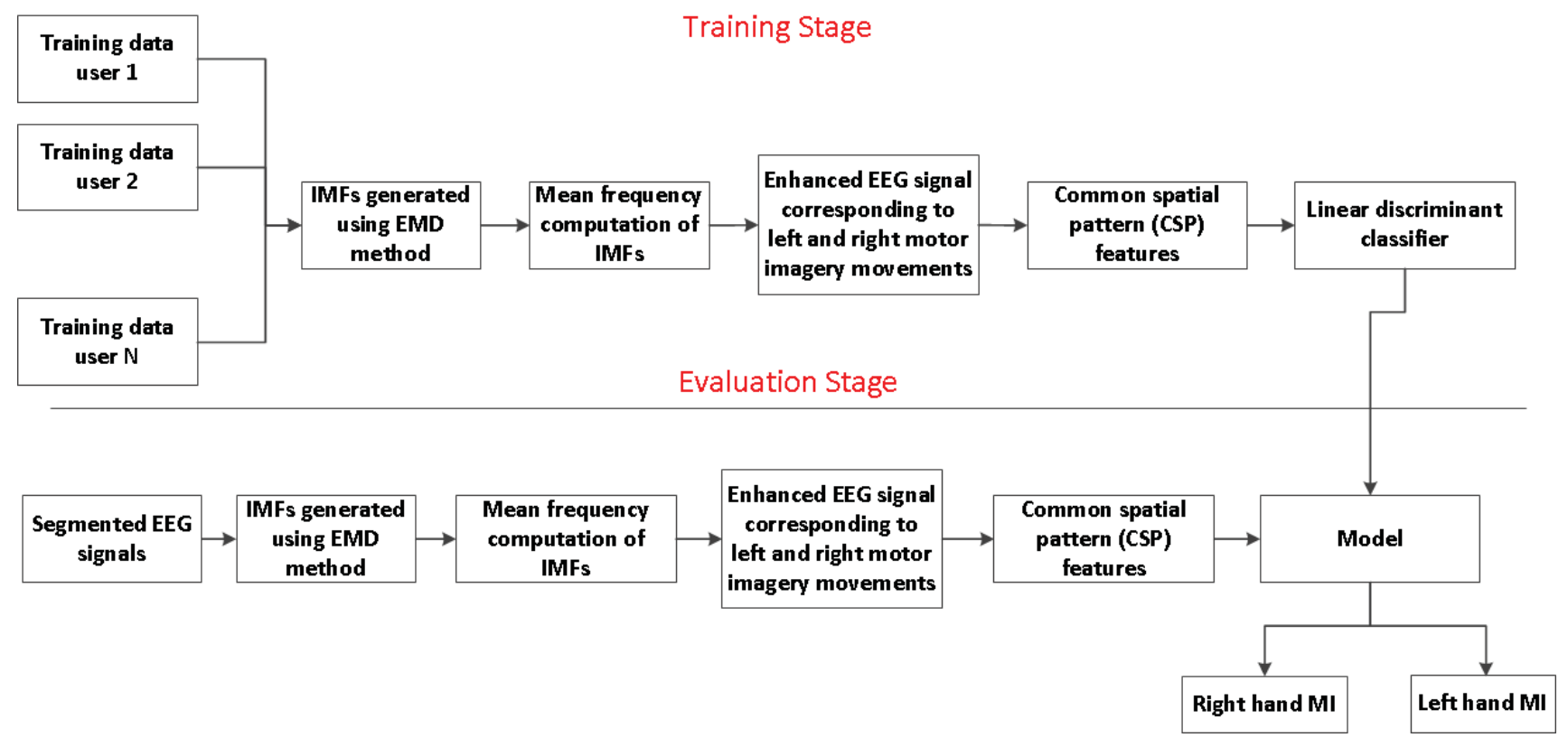

Fig. 1: Block diagram of the proposed method.

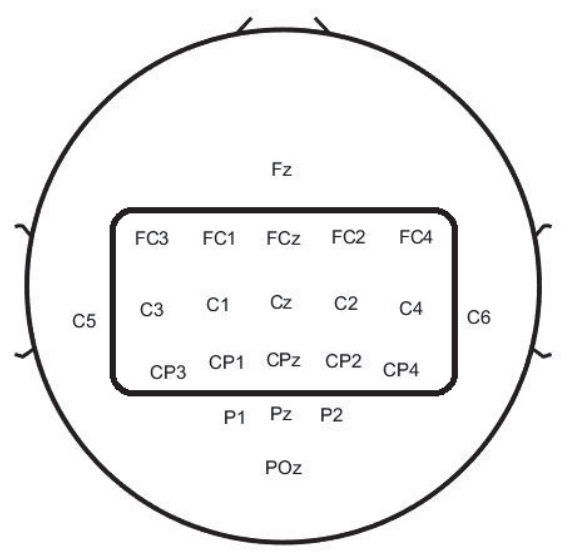

Fig. 2: Headplot showing all the channels locations

$W \in \Re^{C h \times C h}$ where $C h$ represents the number of selected channels. $W$ projects the single trial EEG signal $E \in \Re^{C h \times T}$ in the original sensor space to $Z \in \Re^{C h \times T}$, which is present in the surrogate sensor space, as follows:

$$
P=W \times E
$$

where $E$ is a $C h \times T$ EEG measurement data of a single trial, and $T$ is the number of time points per channel. The rows of $W$ are the spatial filters and the columns of $W^{-1}$ are the common spatial patterns. The spatially filtered signal $P$ given in (3) maximizes the difference in the variance of the two classes. A CSP analysis is applied in order to obtain an effective discrimination of mental states that are characterized by ERD/ERS effects. However, the variances corresponding to only a small number of spatial filters are generally used. The $m$ first and $m$ last rows of $P$ i.e. $P_{t}, t \in\{1,2, \ldots, 2 m\}$ from the feature vector $x_{t}$ given in (3) is input to a classifier. In this study, we have considered $m=4$ and $m=5$. The CSP features of the single trial are then given by:

$$
x_{t}=\log \frac{\operatorname{var}\left(P_{t}\right)}{\sum_{i=1}^{m} \operatorname{var}\left(P_{t}(:, i)\right)+\operatorname{var}\left(P_{t}(:, C h+1-i)\right.}
$$

Then, the CSP based features are extracted to form an input features for LDA classifier.

\section{LINEAR DISCRIMINANT ANALYSIS}

In this paper, we have implemented an LDA classifier which is commonly implemented in EEG-based BCI applications. The LDA classifier tries to reduce the dimensionality and simultaneously protects most of the class discrimination information. Suppose, we have a set of two classes denoted by cls 1 and $\mathrm{cls}_{2}$. Then, we classify the $n$-dimensional sample points $x=\left\{x_{1}, x_{2}, x_{3}, \ldots, x_{n}\right\}, m_{1}$ samples to the class $\mathrm{cls}_{1}$, and $m_{2}$ samples to the class $\mathrm{cls}_{2}$. In this method, we try to enact a line $y=w^{t} x$ from the set of all possible lines. The selected line maximizes the discrimination between the two available classes. For obtaining a good projection vector, we require to measure the distance between the two classes chosen for the study. The mean vector of each class in $x$-space and $y$-space is represented by following equations [18]:

$$
\begin{gathered}
v_{i}=\frac{1}{N_{i}} \sum_{x \in w_{i}} x \\
\text { and } \vartheta_{i}=\frac{1}{N_{i}} \sum_{y \in w_{i}} y=\frac{1}{N_{i}} \sum_{y \in w_{i}} w^{t} x=w^{t} v_{i}
\end{gathered}
$$



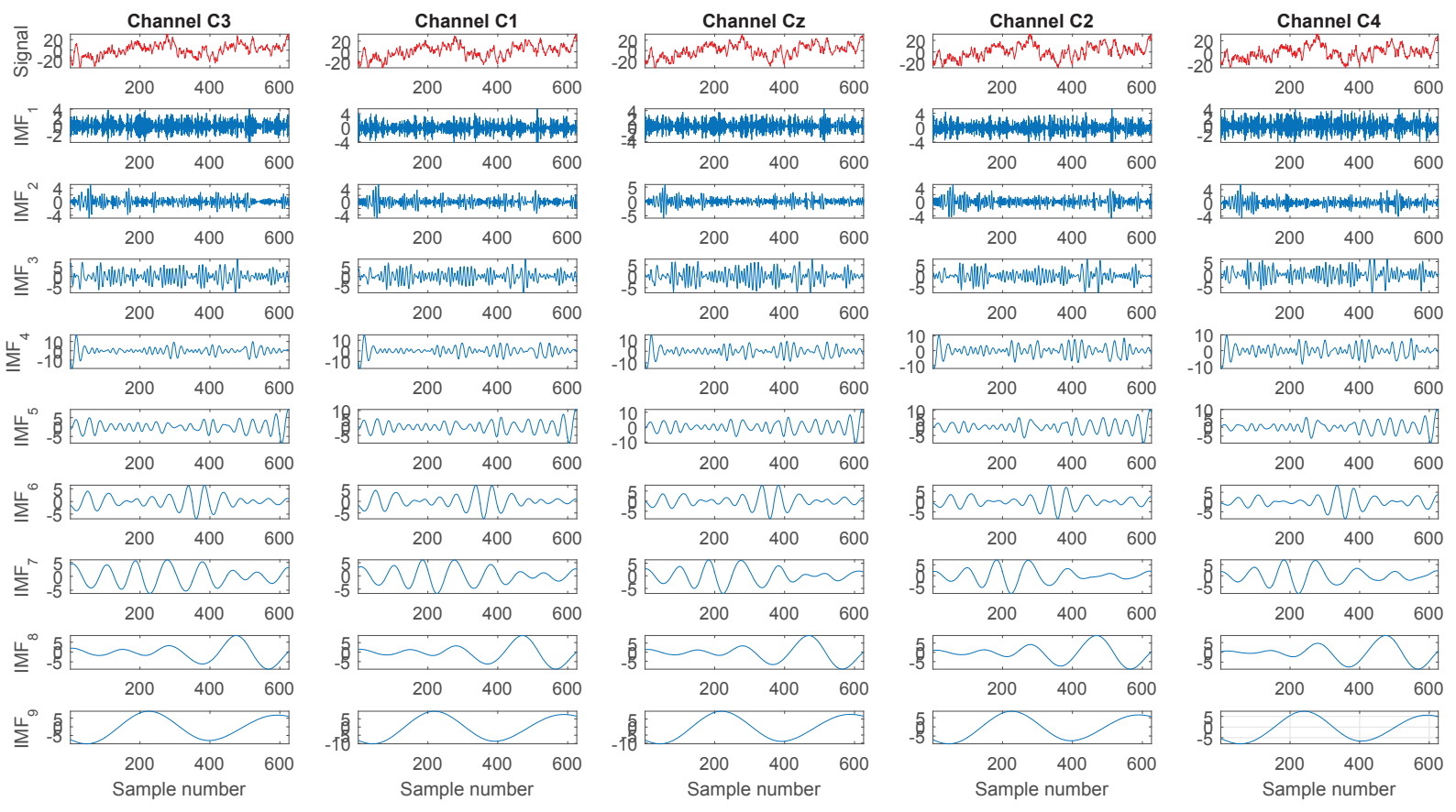

Fig. 3: The EEG signal from $\mathrm{C} 3, \mathrm{C} 1, \mathrm{Cz}, \mathrm{C} 2$ and $\mathrm{C} 4$ of the trial 1 of $\mathrm{A} 01 \mathrm{~T}$ with the first nine IMFs generated for the left hand movement.
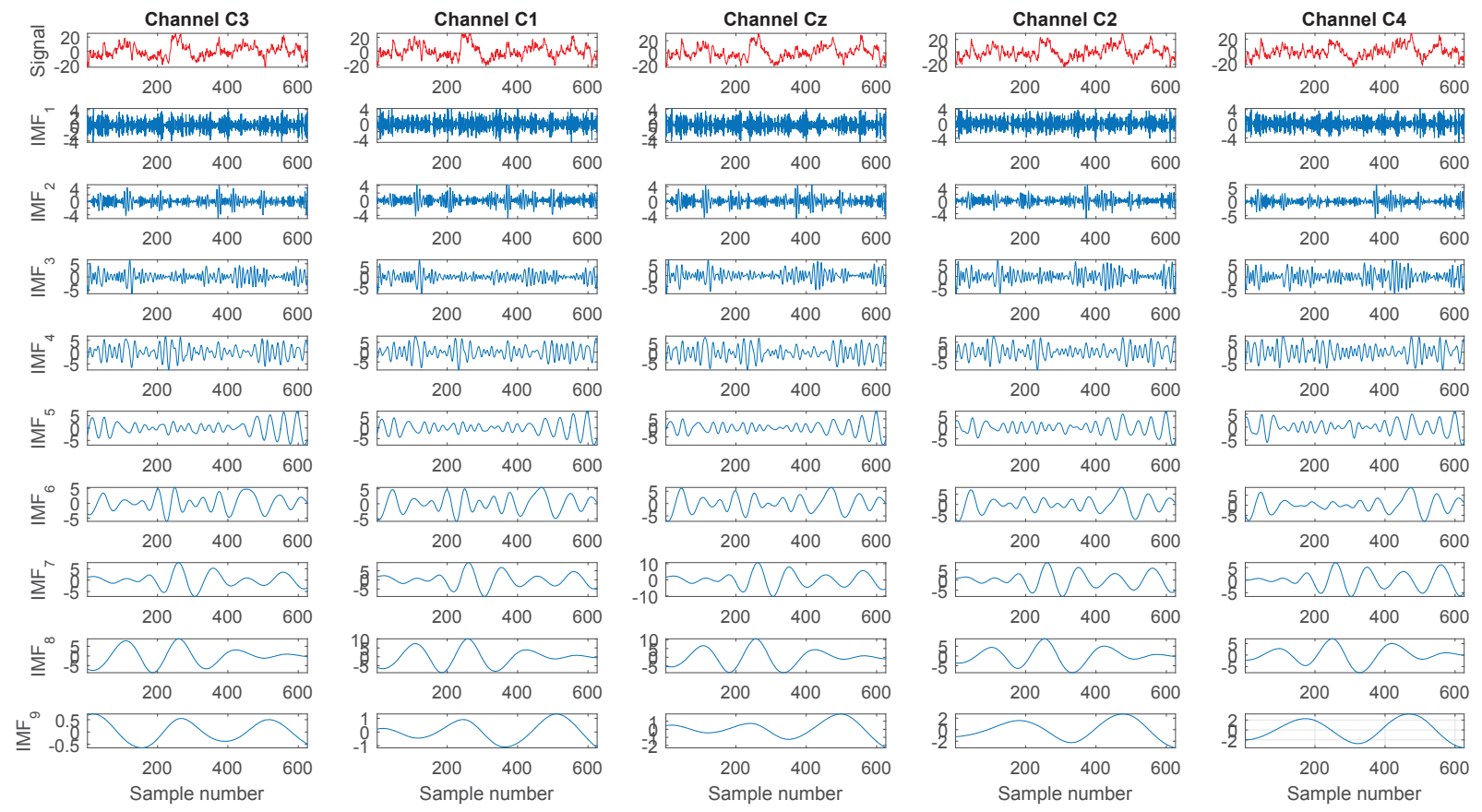

Fig. 4: The EEG signal from $\mathrm{C} 3, \mathrm{C} 1, \mathrm{Cz}, \mathrm{C} 2$ and $\mathrm{C} 4$ of the trial 1 of A01T with the first nine IMFs generated for the right hand movement. 
The objective function is expressed as the distance between the two projected means. It can be defined as follows [18]:

$$
J(w)=\left|\vartheta_{1}-\vartheta_{2}\right|=\left|w^{t}\left(v_{1}-v_{2}\right)\right|
$$

However, the distance measured between projected means may not always be a good measure because the standard deviation between classes has not been considered. In order to overcome this restriction, an enhancement of LDA has been proposed and is known as Fishers LDA classifier. It determines a decision boundary or most likely a hyperplane in the feature space to classify the features in to distinct classes. It finds out the separation boundary between two given distributions in terms of the ratio of two group variances as given below [18], [19]:

$$
J(w)=\frac{\sigma_{\text {between }}^{2}}{\sigma_{\text {within }}^{2}}=\frac{w^{t}\left(v_{1}-v_{2}\right)^{2}}{w^{t} S_{1} w+w^{t} S_{2} w}
$$

where $v_{1}, v_{2}$ are the mean of the classes and $S_{1}, S_{2}$ are the variances of the feature distributions between two classes $w_{1}, w_{2}$ respectively. The maximum separation between two classes can be shown by (9) as [18]:

$$
w^{*}=\left(S_{1}+S_{2}\right)^{-1}\left(v_{1}-v_{2}\right)
$$

The $w^{*}$ is weight vector which provides optimum direction of projection of the data. In Fishers LDA, the decision boundary uses following equation to classify the feature vector $D(m)$ as:

$$
P(m)=D(m) w^{t}+b
$$

where $b$ is the bias or threshold. The features are assigned to one of the classes based on the sign of the $P(m)$.

\section{RESULTS AND DISCUSSION}

The proposed MEMD-SI-BCI based filtering has been evaluated on publicly available BCI competition IV dataset $2 \mathrm{~A}$ [13]. The dataset contains EEG signals from nine healthy subjects, denoted by A01-A09. Each subject contains one training and one evaluation session. In this study, we have considered EEG signals recorded from fifteen channels in Fig. 2 related to the sensorimotor areas from all nine subjects to demonstrate the effectiveness of the proposed method. The data were recorded from twenty bipolar channels and three EOG channels with a sampling frequency of $250 \mathrm{~Hz}$.

There are seventy two number of trials provided in each session. Each trial involved a paradigm period of 7.5 second [30]. In training stage, a single session namely ${ }^{\wedge} T$ has been used. For the evaluation phase, we have used one session namely, ${ }^{\wedge} E$ for computing the accuracy in the classification of left and right MI EEG signals. It should be noted that, the ${ }^{\wedge}$ in the session name denotes the subject number which ranges from A01 to A09.

In order to compute the classification accuracy (in \%) in the training stage, we have applied 5-fold cross validation. In the evaluation session, the model has been trained with LDA classifier using $100 \%$ data from the A $0 \beta \mathrm{T}$ and evaluated on the $100 \%$ data for the session, $\mathrm{A} 0 \beta \mathrm{E}$, where $\beta$ denotes the subject number. Since the MI task starts at 3 second, we have extracted the feature in both of the training and evaluation sessions corresponding to EEG signals from 0.5 second to 2.5 second time-interval after the start of MI paradigm. We have computed the classification accuracy using LDA classifier for two class classification of the left and right hand MI EEG signals in both training and evaluation sessions for each of the subjects.

In order to explain the working of the MEMD method, we have considered two single trial EEG signals from the dataset A01T fifteen channels signals to obtain IMFs but we have shown the plot for five channels. The left MI EEG signal from channels and its first nine IMFs are shown in Fig. 3. Similarly, the Fig. 4 depicts the right hand MI EEG signal from the five channels and its first nine IMFs.

The statistical measure, namely, mean frequency has been calculated for each multivarite IMFs of the EEG signals from the selected fifteen channels in the motor cortex region corresponding to left and right hand MI tasks. To achieve enhanced EEG signals corresponding to left and right hand MI tasks, the IMFs whose mean frequencies fall in the range 6$24 \mathrm{~Hz}$ were selected. This frequency range takes into account the $\mu$ band $(8-13 \mathrm{~Hz})$ and low $\beta$ band $(18-24 \mathrm{~Hz})$. These frequency bands have considerable importance in order to classify left and right hand MI EEG signals. The CSP feature is then computed for the enhanced EEG signals obtained using the selected IMFs. In our study we have reported the results obtained using two spatial filters $m=4$ and $m=5$ where $m$ denotes the the first $m$ and the last $m$ columns of spatial filter matrix. Then, the extracted feature has been fed to as an input feature to the LDA classifier for classification of left and right hand MI EEG signals.

Table I presents the classification accuracy with MEMD based filtering-SI-BCI (MEMD-SI-BCI) and with the raw SI-BCI method for BCI competition IV dataset $2 \mathrm{~A}$. The method has provided the enhanced EEG signals using subject independent MEMD-BCI for the each of the nine subjects in both training $T$ and evaluation $E$ sessions respectively. In this work, only fifteen channels corresponding to motor cortex have been considered of the provided twenty two channels for obtaining the results.

Comparing the MEMD-SI-BCI results, it is clear that the new method presented in this paper provides a significant improvement in classification accuracy in evaluation session of all nine subjects when compared with the with the raw SIBCI results. It has shown improvement $>11 \%(p<0.001)$ in evaluation session with $m=4$ and $>11 \%(p<0.001)$ in evaluation session with $m=5$. In the training session, since we have created generalized model for all the subjects, there is slight improvement in the classification accuracy. The nine of the nine subjects have shown improvement in the evaluation stage with $m=4$. A total of seven out of nine subjects have shown highly significant improvement $>10 \%$ and the other two subjects have shown improvement $>2 \%$. On the other hand, with $m=5$, two of the subjects have shown significant improvement of $>20 \%$ and a total of seven subjects 
TABLE I: CLASSIFICATION ACCURACIES USING THE PROPOSED METHOD BASED ON MEMD AND WITHOUT MEMD STUDIED ON BCI COMPETITION IV DATASET 2A

\begin{tabular}{|c|c|c|c|c|c|c|c|c|}
\hline \multirow[b]{2}{*}{ Subject } & \multicolumn{4}{|c|}{ Accuracy with MEMD-SI-BCI } & \multicolumn{4}{|c|}{ Accuracy with SI-BCI } \\
\hline & Training & $\begin{array}{l}\text { Evaluation } \\
=4\end{array}$ & Training & $\begin{array}{l}\text { Evaluation } \\
=5\end{array}$ & Training & $\begin{array}{l}\text { Evaluation } \\
=4\end{array}$ & Training & $\begin{array}{l}\text { Evaluation } \\
=5\end{array}$ \\
\hline A01 & 70.14 & 91.67 & 72.16 & 92.36 & 68.2 & 69.44 & 68.2 & 68.06 \\
\hline A02 & 70.21 & 55.56 & 71.07 & 58.33 & 67.91 & 49.31 & 67.9 & 52.78 \\
\hline A03 & 69.22 & 90.97 & 67.45 & 91.67 & 68.36 & 70.83 & 68.14 & 71.53 \\
\hline A04 & 70.6 & 62.5 & 70.68 & 63.89 & 68.21 & 59.72 & 67.91 & 58.33 \\
\hline A05 & 68.91 & 61.11 & 71.45 & 59.03 & 68.29 & 49.31 & 68.52 & 49.31 \\
\hline A06 & 70.37 & 68.06 & 71.29 & 67.36 & 68.44 & 55.56 & 68.68 & 55.56 \\
\hline A07 & 70.29 & 61.11 & 70.37 & 60.42 & 68.3 & 50.69 & 67.98 & 51.39 \\
\hline A08 & 70.52 & 96.53 & 70.67 & 96.53 & 68.29 & 91.67 & 69.14 & 91.67 \\
\hline A09 & 65.44 & 65.97 & 67.82 & 66.67 & 67.9 & 54.86 & 68.36 & 56.94 \\
\hline Average & 69.52 & 72.61 & 70.33 & 72.92 & 68.21 & 61.27 & 68.31 & 61.73 \\
\hline Std & 1.64 & 15.79 & 1.62 & 15.81 & 0.19 & 13.95 & 0.41 & 13.45 \\
\hline$p$-value & 0.034 & 0.001 & 0.005 & 0.001 & & & & \\
\hline
\end{tabular}

have shown improvement in the range of $>4 \%$ and $<24 \%$. To conclude, with $m=4$ and $m=5$, we have achieved improvement in all of the nine subjects in the evaluation sessions. The $p$-values have been calculated using the anova2 command available in MATLAB.

\section{CONCLUSION}

We have explored an application of the multivariate empirical mode decomposition based filtering method using subject independent design to achieve high performance in motor imagery based brain-computer interface. A group of IMFs whose mean frequencies fall in the frequency range of $\mu$ and $\beta$ rhythms have provided significant improvement in terms of classification accuracy to classify the left and right hand MI EEG signals when compared without using the MEMD based filtering method. In future, it would be of interest to propose new features based on the MEMD method and connectivity analysis method to classify the MI EEG signals. With the proposed method, enhanced feature separability has been achieved using MEMD based filtering using subject independent method resulting in low classification errors. It has helped to reduce the performance deterioration due to EEG non-stationarities to some extent in evaluation stage. Adaptive classification techniques can be explored with the present design to handle the non-stationarities more effectively

\section{ACKNOWLEDGMENT}

P.G. was supported by Ulster University Vice-Chancellors research scholarship (VCRS). G.P. was supported by the Northern Ireland Functional Brain Mapping Facility project (1303/101154803), and also by the UKIERI DST Thematic Partnership project "A BCI operated hand exoskeleton based neuro-rehabilitation system" (UKIERI-DST-201314/126). During the work reported in the paper, R.B.P. was a visiting scholar at ISRC Ulster University with funding support from the Northern Ireland Functional Brain Mapping Facility project (1303/101154803).

\section{REFERENCES}

[1] G. Pfurtscheller, C. Neuper, D. Flotzinger, and M. Pregenzer, "EEGbased discrimination between imagination of right and left hand movement," Electroencephalography and Clinical Neurophysiology, vol. 103, no. 6, pp. 642-651, 1997.

[2] G. Pfurtscheller and C. Neuper, "Motor imagery and direct braincomputer interface," in Proc. IEEE. v89, 2001, pp. 1123-1134.

[3] S. Shahid and G. Prasad, "Bispectrum-based feature extraction technique for devising a practical brain-computer interface," Journal of Neural Engineering, vol. 8, no. 2, p. 025014, 2011.

[4] R. Sharma and R. B. Pachori, "Classification of epileptic seizures in EEG signals based on phase space representation of intrinsic mode functions," Expert Systems with Applications, vol. 42, no. 3, pp. 1106-1117, 2015.

[5] G. Pfurtscheller, C. Brunner, A. Schlögl, and F. L. Da Silva, "Mu rhythm (de) synchronization and EEG single-trial classification of different motor imagery tasks," Neuroimage, vol. 31, no. 1, pp. 153-159, 2006.

[6] F. Lotte, C. Guan, and K. K. Ang, "Comparison of designs towards a subject-independent brain-computer interface based on motor imagery," in Engineering in Medicine and Biology Society, 2009. EMBC 2009. Annual International Conference of the IEEE. IEEE, 2009, pp. $4543-$ 4546.

[7] B. Reuderink, J. Farquhar, M. Poel, and A. Nijholt, "A subjectindependent brain-computer interface based on smoothed, second-order baselining," in Engineering in medicine and biology society, EMBC, 2011 annual international conference of the IEEE. IEEE, 2011, pp. 4600-4604.

[8] P. Gaur, R. B. Pachori, H. Wang, and G. Prasad, "An empirical mode decomposition based filtering method for classification of motor-imagery EEG signals for enhancing brain-computer interface," in International Joint Conference on Neural Networks, 2015, pp. 1-7.

[9] C. Park, D. Looney, A. Ahrabian, D. P. Mandic et al., "Classification of motor imagery BCI using multivariate empirical mode decomposition," IEEE Transactions on Neural Systems and Rehabilitation Engineering, vol. 21 , no. 1, pp. 10-22, 2013.

[10] D. Coyle, G. Prasad, and T. M. McGinnity, "A time-frequency approach to feature extraction for a brain-computer interface with a comparative analysis of performance measures," EURASIP Journal on Applied Signal Processing, vol. 2005, pp. 3141-3151, 2005.

[11] K. K. Ang, Z. Y. Chin, C. Wang, C. Guan, and H. Zhang, "Filter bank common spatial pattern algorithm on BCI competition IV datasets $2 \mathrm{a}$ and 2b," Frontiers in Neuroscience, vol. 6, 2012.

[12] H. Zhang, H. Yang, and C. Guan, "Bayesian learning for spatial filtering in an EEG-based brain-computer interface," IEEE Transactions on Neural Networks and Learning Systems, vol. 24, no. 7, pp. 1049-1060, 2013.

[13] C. Brunner, R. Leeb, G. Müller-Putz, A. Schlögl, and G. Pfurtscheller, "BCI Competition 2008-Graz data set A," Institute for Knowledge Discovery (Laboratory of Brain-Computer Interfaces), Graz University of Technology, pp. 136-142, 2008.

[14] N. E. Huang, Z. Shen, S. R. Long, M. C. Wu, H. H. Shih, Q. Zheng, N.C. Yen, C. C. Tung, and H. H. Liu, "The empirical mode decomposition and the Hilbert spectrum for nonlinear and non-stationary time series 
analysis," in Proceedings of the Royal Society of London A: Mathematical, Physical and Engineering Sciences, vol. 454, no. 1971. The Royal Society, 1998, pp. 903-995.

[15] Z. Wu and N. E. Huang, "Ensemble empirical mode decomposition: a noise-assisted data analysis method," Advances in Adaptive Data Analysis, vol. 1, no. 01, pp. 1-41, 2009.

[16] N. ur Rehman, C. Park, N. E. Huang, and D. P. Mandic, "EMD via MEMD: multivariate noise-aided computation of standard EMD," Advances in Adaptive Data Analysis, vol. 5, no. 02, p. 1350007, 2013.

[17] B. Blankertz, R. Tomioka, S. Lemm, M. Kawanabe, and K.-R. Muller, "Optimizing spatial filters for robust EEG single-trial analysis," Signal Processing Magazine, IEEE, vol. 25, no. 1, pp. 41-56, 2008.

[18] F. Lotte, M. Congedo, A. Lécuyer, and F. Lamarche, "A review of classification algorithms for EEG-based brain-computer interfaces," Journal of Neural Engineering, vol. 4, 2007.

[19] C. Vidaurre, M. Kawanabe, P. Von Bunau, B. Blankertz, and K. Muller, "Toward unsupervised adaptation of LDA for brain-computer interfaces," IEEE Transactions on Biomedical Engineering, vol. 58, no. 3, pp. 587-597, 2011. 